\title{
The Effects of Divided Attention and Selective Attention on the Accuracy of Memory
}

\author{
Zhuxuan $\mathrm{Ji}^{1, *}, \dagger$, Hong Huang ${ }^{2, \dagger}$, Zizhan Wang ${ }^{3, \dagger}$ \\ ${ }^{1}$ All Saints Anglican School, Gold Coast, 4213, Australia \\ ${ }^{2}$ Quanzhou No.5 HighSchool, Quanzhou, Fujian, China \\ ${ }^{3}$ Xi'an Gaoxin NO.1 High School Fengdong High school, Xi'an, China \\ *Corresponding author. Email: 020554@asas.qld.edu.au \\ $\dagger$ These authors contributed equally.
}

\begin{abstract}
Previous research has shown that memory accuracy can be affected by distracting attention. Therefore, this study aimed to explore the effects of different types of attention during the encoding stage of memory on memory accuracy (the accuracy of recalled words). Sixteen (eight male and eight female) senior school students were randomly and equally assigned to an experimental group (divided attention group) and a control group (selective attention group). Listening to music as a popular interference is utilized to divide an individual's attention in this experiment. Both selective and divided attention group participants were asked to memorize several English words in a certain period. However, the divided attention group participants were set to have an interference while listening to the words, whereas there was no interference in the selective attention group. Compared to the control group, the selective attention on words correctly recalling had a significantly higher mean score than the mean score of the divided attention $(\mathrm{p}<0.05)$. This finding suggests that the effects of the divided attention during the encoding stage on the accuracy of retrieved memory are negative compared to selective attention.
\end{abstract}

Keywords: Divided attention, Selective attention, Memory

\section{INTRODUCTION}

Attention can actively process specific information in the environment while tuning out other details [1]. In psychologist and philosopher William James's 1890 book "The Principles of Psychology", he wrote that attention was the taking possession by the mind, in clear and vivid form, of one out of what may seem several simultaneously possible objects or trains of thought. It implies withdrawal from some things to deal effectively with others." [2]

Selective attention (SA) is the process of directing our consciousness to relevant stimuli while ignoring irrelevant stimuli in the environment. This is an important process because we can process only so much information in a given amount of time. Selective attention allows us to ignore insignificant details and focus on what is important. [3-6] However, divided attention (DA) can integrate into multiple parallel stimuli. When our brain performs the divided attention, our attention is divided into different parts to achieve different tasks, rather than alternating between them $[1$,
7]. Therefore, divided attention may appear to be the efficient way to complete two tasks simultaneously, but in fact, it will affect the efficiency with which each task is completed. Furthermore, this also leads to the accuracy and precision at which tasks are completed be lacking. Memory refers to the processes used to acquire, store, retain, and later retrieve information. There are three major processes involved in memory: encoding, storage, and retrieval $[8,9]$. When attention is distracted during retrieval, interference occurs only when memory and simultaneous tasks compete to enter the representation system of a particular word; no such specificity is necessary to create interference at encoding [5]. However, the experiment conducted by Craik et al. concluded that division of attention was associated with a much greater drop in memory performance when attention was divided at encoding than at retrieval [10]. In other words, Craik's study suggested SA and DA both have impacts on the encoding stage. Therefore, to test the accuracy of these two literatures. Based on Craik's experiment in 1996 and his experiment in 2018, a new experiment will be 
extended in this study to investigate whether divided attention has an effect on memory in the encoding phase.

The results from Craik's 1996 study are not reliable at present as individuals in the 20th century will have different strengths of memory than individuals in the 21st century. Therefore, an experiment of Craik i018 was also referred to in the current experiment.

The experiment conducted by Craik et al. was primarily focused on finding the effects of divided attention (DA) on different processes in human memory [10]. The effects of divided attention are all measured and recorded when the encoding and retrieval process is investigated under free recall, cued recall, and recognition memory. First, the experiment required participants to be presented with a 60 s memory task. After this, to eliminate the recency effect, they were required to complete a math task. Finally, participants orally recalled the 15 words as many as they could in their 30s during the recall phase. To divide participants' attention, a reaction time task (RT) was performed during the memory task, the recall phase, during both or separately (control). Through the analysis of the result data, during the encoding stage, divided attention led to a large increase in memory performance and a small increase in reaction time; and this resulted in a small decrease in-memory performance and a large increase in reaction time during the recall stage. Therefore, based on this discovery, the researchers concluded that divided attention had a greater impact on the encoding process as memory performance changes in task emphasis were more significant during the encoding stage than during the retrieval stage.

Furthermore, Craik's 2018 study provided further evidence, extended from his 1996 study [11]. Both studies were sought to find out the effects of divided attention at encoding and retrieval. However, the 2018 study improved the experiment's methodology, which led to more accurate results than the 1996 study.

The current study aims to explore whether the divided attention affects the accuracy of memory through modifying the original experiment by the Craik et al. (1996) study. Specifically, a more common form of divided attention in life (listening to music) is utilized in this experiment rather than doing the reaction time task. Because many students like to listen to music while they are doing their homework or reading. Therefore, the results obtained from this experiment are highly useful for students understanding how listening to music affects encoding memory and how it limits or contributes to an individual's ability to encode memory.

\section{METHOD}

\subsection{Participants}

The participants included a sample selected from 400 students aged 17-19 attending a high school in Queensland, Australia. To recruit participants, a convenience sampling method was utilized. The sample consisted of 16 (males $=8$, females $=8$ ) grade 12 students, aged 18 years old, and participants were randomly and equally assigned to one of two conditions. All the participants are used to listen to music while doing their homework or reading books. They were randomly divided into two groups, and each group has 4 males and 4 females. One group was under the divided attention (DA) group, while the other group was under the selective attention (SA) group.

All the participants have similar intelligence quotients, normal mental and similar Chinese and English skills. The participants' first language is Chinese, and their second language is English. All participants were received informed consent aware of the meaning of the experiment, and agreed to be the volunteers. The experiment method was briefed before it began, and participants were informed what the collected results would be used for and that they always have the freedom to withdraw the data at any time.

\subsection{Materials}

The design and the implementation of two experiments were conducted by utilizing the psychopy. For each group, after watching the slide of the introduction of the experiment on psychopy, a list of 9 English words was presented in white on the black background. The 9 words were selected randomly from the SAT vocabulary book, with no similar meanings. However, each word is similar in length, with an average of seven letters. The word list was presented for 120 s (each participant had about13s to remember each word). For the SA group, the word list was presented alone. In contrast, for the DA group, the word list was presented with the pure piano song (composed by Alisa), and the piano song was played for the 90s, after the $90 \mathrm{~s}$, the time of memorizing time, and the song stopped at the same time.

\subsection{Procedure}

All the participants were required to take the experiment where they often studied and remembered things and were familiar with. The time the participants took part in the experiments was chosen by themselves, which was also the time suitable for them to study and remember things. And to make sure the participants did not cheat or be interfered with by others, they were required to turn on the cameras to be monitored. 
The two groups of participants entered the experiment and read the introduction of the experiment. After 10s, the experiment began. The participants saw the word list of 9 words, while the participants in the DA group also heard the song. After the 120s, the word list and the piano song disappeared immediately for themselves to remember by their own methods. The participants then are asked to take the free-response tasks, in which they should type down all they remember about the 9 words in the 30s, and their results would be tested the accuracy to the real word list, and 10s later, the tasks began. After this, participants saw a blank screen, and they could type down there to show what they remembered during the 30s. During the typing phase, the participants were monitored by the cameras. After the 30s, all that the participants typed down on the blank screen was recorded and disappeared, and the next slide showed them the task was finished, then the participants click the certain button on the keyboard to quit the experiment.

\section{RESULTS}

\subsection{Descriptive statistics}

There were no obvious outliers identified from the raw data, and the data was considered as a ratio as it included true zero. Therefore, the most appropriate measure of central tendency is chosen as the mean and the standard deviation as the measure of dispersion. Descriptive statistics are displayed in Table 1 and Figure 1.

Table 1. Mean, standard deviation (SD), and standard error of mean (SEM) scores for the proportion of words correctly recalled in selective attention (SA) and divided attention (DA) conditions.

\begin{tabular}{lll}
\hline & Selective attention & Divided attention \\
\hline Mean & 0. & 0.19 \\
SD & 52 & 0.13 \\
SE & 0.13 & 0.05 \\
& 0.05 & \\
\hline
\end{tabular}

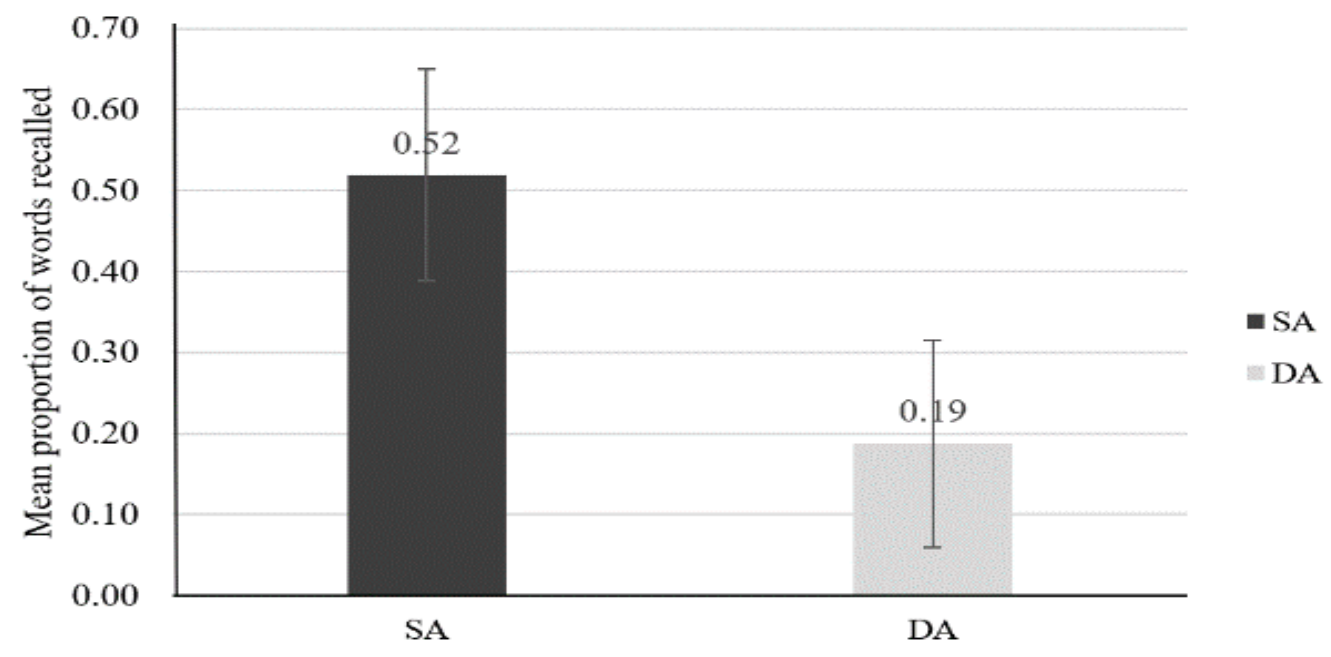

Figure 1 Mean proportion of words correctly recalled, in selective attention (SA) and divided attention (DA) conditions. Error bars represent standard deviation.

\subsection{Trends}

The results show that the selective attention (SA) on words correctly recalling has a significantly higher mean score $(0.52)$ than the mean score $(0.19)$ of the divided attention (DA). The standard deviation (s) in the SA group (0.13) has an equal dispersion score to the DA group (0.13), which indicates the SA group and DA group have the same variability between participants. Error bars represent standard deviation (Figure 1).
Figure 1 suggests that the results didn't fall within the same range for both conditions as the error bars are not overlapping.

\subsection{Statistical test}

The data is not normally distributed due to the small sample size of each group $(n=8)$. Therefore, a MannWhitney $U$ was used as the most appropriate nonparametric technique to analyse the data as the data was 
ratio; the research design utilized independent groups; the experiment sought to determine a relationship between SA and DA on recalling method; the variance was equal $(\mathrm{SA} \mathrm{s}=0.13$, DA $\mathrm{s}=0.13)$.

\section{DISCUSSION}

The purpose of our study was to explore whether the implementation of memory tasks in the case of distraction will increase the proportion of words that can be correctly recalled from the list compared with selective attention.

The present study supports the idea that the results show that the selective attention (SA) on words correctly recalling has a much higher mean score $(0.52)$ than the mean score (0.19) of the divided attention (DA). The standard deviation (s) in the SA group (0.13) has an equal dispersion score to the DA group (0.13), which indicates the SA group and DA group have the same variability between participants.

A possible explanation for this might be that in answer to the research question, "Does performing a memory task under divided attention increase the proportion of words that can be correctly recalled from the list compared to selective attention?" The p-value was $0.002(\leq .05)$, which represented a statistically significant difference between the means of two conditions (SA and DA). The results also showed that the selective attention (SA) on words correctly recalling has a much higher mean score $(0.52)$ than the mean score $(0.19)$ of the divided attention (DA). Therefore, there would be a significant difference between the level of attention and the proportion of words correctly recalled.

Like any other study, this study is not free from limitations. In conclusion, our research provides some convincing preliminary evidence for exploring SA and DA. However, further work is required in several areas. These can be explained as a lack of reliability and validity in the experimental process.

Both conditions (SA and DA) were observed with a large standard deviation. A large standard deviation represents the data's wide dispersion around the mean, which suggests that the extraneous variables in this experiment were not fully controlled. Therefore, the data is less reliable. However, the non-overlapping standard deviation error bars, presented in Figure 1., suggest the dispersion of the data is small, and means have a significant difference, making the data more reliable.

The wide dispersion of data indicates that the experimental process is unreliable. Although the experiment attempted to control extraneous variables, some uncertainty was observed due to participants' nature. In the process of trying to control for extraneous variables, participants are randomly assigned to each condition. However, the small sample size $(n=8)$, the convenience sampling, and the independent grouping design were also likely to result in the dispersion of data, leading to the unreliability of results.

Further consideration could be given to the effect of several steps of the experimental process on the reliability of the results. In this experiment, the task of eliminating the recency effect was not appropriate as it required participants to find words on a paper, which might divide the participants' attention again. Therefore, tasks in the process of eliminating recency effects cannot be related to the divided attention tasks, which could affect the reliability of the experimental methodology and lead to unreliable results.

The population validity is considered to be low as the small sample size $(\mathrm{N}=16)$ and the results from the unrepresentative sample (aged 18 years old teenagers) would not resemble the results from the general population (adults). In addition, as the entire experiment was conducted in a school laboratory with higher controls for variables than in most situations, the ecological validity is also considered to be low. Therefore, it's problematic to attempt to generalize the results to the real world.

This research also has a number of strengths worth mentioning. Through analysis of the data in the experiment, the results showed that there was a significant difference in the accuracy of recalling between divided attention and selective attention. However, the results also indicated that the evidence still had noticeable limitations and uncertainties. To improve the reliability and validity of the experiment methodology, the following improvements and extensions are suggested.

To improve the experiment's reliability, it is an effective improvement to increase the sample size $(\geq 100)$, which helps to reduce the dispersion of the data. A further improvement of the experiment is to use a paired participant design in which participants complete a predictive test that tested their memory ability before participating in the experiment. Then, participants with the same memory level are randomly and equally assigned to each condition, which could reduce the impact of natural participant variability on the experiment's reliability.

In terms of improving the population validity, an approach is to use random or stratified-random sampling techniques, which can increase the representativeness of the population through samples, instead of using convenience sampling.

An extension of the experiment could be to explore the effect of word complexity on word recall: whether longer, more complex words decrease the number of words that can be recalled. 


\section{CONCLUSION}

The present study explored the effects of divided attention and selective attention on the accuracy of memory. The results showed that selective attention (SA) on words correctly recalling has a much higher mean score than divided attention (DA). Despite the above limitation, this study demonstrates that memorizing words while listening to music affects the accuracy of word spelling during recall. Further confirming that the divided attention during the encoding stage reduces the accuracy of retrieved memory compared to the selective attention. The results can also be applied to normal life. For example, listening to music while doing homework affects the accuracy of homework. In addition, the habit of listening to music while working in daily work has also been proved that it is more likely to reduce the overall work efficiency. Therefore, based on the above applications, this research can play an important role in improving work efficiency.

\section{REFERENCES}

[1] Fernandes MA (2000). Divided attention and memory: Evidence of substantial interference effects at retrieval and encoding. Journal of experimental psychology, General, 129(2). doi:10.1037//0096-3445.129.2.155

[2] James, W. (1890). The principles of psychology, Vol. 2. Henry Holt and Company.

[3] Treisman, A. M. (1964). Selective attention in man. British medical bulletin, 20(1), 12-16.

[4] Treisman, A. M. (1969). Strategies and models of selective attention. Psychological review, 76(3), 282 .

[5] Luck, S. J., \&Kappenman, E. S. (2012). ERP components and selective attention.

[6] Mirza, M. B., Adams, R. A., Friston, K., \& Parr, T. (2019). Introducing a Bayesian model of selective attention based on active inference. Scientific reports, 9(1), 1-22.

[7] Salo, E., Salmela, V., Salmi, J., Numminen, J., \&Alho, K. (2017). Brain activity associated with selective attention, divided attention and distraction. Brain research, 1664, 25-36.

[8] Corbetta, M., Miezin, F. M., Dobmeyer, S., Shulman, G. L., \& Petersen, S. E. (1991). Selective and divided attention during visual discriminations of shape, color, and speed: functional anatomy by positron emission tomography. Journal of neuroscience, 11(8), 2383-2402.
[9] Hahn, B., Wolkenberg, F. A., Ross, T. J., Myers, C. S., Heishman, S. J., Stein, D. J., ... \& Stein, E. A. (2008). Divided versus selective attention: Evidence for common processing mechanisms. Brain research, 1215, 137-146.

[10] Craik, F. I., Govoni, R., Naveh-Benjamin, M., \& Anderson, N. D. (1996). The effects of divided attention on encoding and retrieval processes in human memory. Journal of Experimental Psychology: General, 125(2), 159.

[11] Craik, F. I., Eftekhari, E., \&Binns, M. A. (2018). Effects of divided attention at encoding and retrieval: Further data. Memory \& cognition, 46(8), 1263-1277. 